\title{
Hat Kunst eine Konfession? Ein Schreibgespräch
}

\section{David Plüss und Johannes StüCKelberger}

David Plüss: Im Rahmen des Forschungsprojekts Holyspace, Holyways an der Hochschule Luzern haben wir wiederholt die katholische Prägung von in der Innerschweiz entstandener Kunst festgestellt. ${ }^{1}$ Die konfessionellen Konturen der erforschten Künstlerinnen und Künstler, Bilder und Installationen beziehen sich zunächst - auf der Oberfläche gewissermaßen - auf die eingespielten Traditionsbezüge, auf die verwendete Symbolik und die Ikonografie. Kruzifixe, Schutzmantelmadonnen und Heilige lösen selbst bei uns spätmodernen Protestanten leichtes Befremden aus. Eine mir emotional und biografisch unvertraute Welt wird hier künstlerisch in Szene gesetzt. Davon ausgehend stellt sich mir die Frage nach der Konfessionalität von Kunst, vor allem dann, wenn sie sich auf religiöse Symbolik bezieht. Ist es überhaupt sinnvoll, das Verhältnis von Religion und Kunst außerhalb der Konfessionsfrage zu verhandeln? Stellt sich das Verhältnis nicht als ein je anderes dar, sobald unterschiedliche Religionskulturen ins Spiel kommen? Ich meine tatsächlich, Kunst habe eine Konfession - in diesem weiten Verständnis. Jedenfalls Kunst mit einem explizit religiösen Hintergrund. Vielleicht aber alle Kunst, auch nicht-religiöse.

Johannes Stückelberger: Die Frage nach der konfessionellen Prägung der Kunst beschäftigt mich seit langem. ${ }^{2}$ Auch ich stelle fest, dass nicht nur der religiösen, sondern oftmals auch der nicht-religiösen Kunst - ohne letztere damit religiös vereinnahmen zu wollen - bestimmte Gottesbilder zugrunde liegen, die in der Regel konfessionell geprägt sind. Im Zusammenhang mit moderner Kunst

1 Zum Forschungsprojekt Holyspace, Holyways siehe: http://holy.kunstforschunglu zern.ch/ (ges. am 24.4.2012).

2 Vgl. Stückelberger, Johannes: Das unsichtbare Bild - Prolegomena zu einer reformierten Ästhetik, in: Krieg, Matthias, Rüsch, Martin, Stückelberger, Johannes, Zeindler, Matthias (Hg.): Das unsichtbare Bild. Die Ästhetik des Bilderverbotes, Zürich (TVZ) 2005, S. 11-19; Stückelberger, Johannes: Das unsichtbare Bild als Figur protestantischer Ästhetik, in: Theologische Zeitschrift, 2/67, Basel (Reinhardt), 2011, S. 203-222. 
spreche ich gerne von ,latenten Gottesbildern‘3 , da der religiöse Bezug oft verborgen bleibt oder nicht so offensichtlich ist.

Wir sind beide reformiert. Es soll in unserem Gespräch jedoch nicht darum gehen, reformiert und katholisch geprägte Kunst gegeneinander auszuspielen. Ich sehe unseren Beitrag als Chance, in einem Forschungsprojekt, in dem vor allem in einem katholischen Umfeld entstandene Kunst zur Sprache kommt, die Frage der konfessionellen Prägung auch aus einer protestantischen Perspektive zu stellen. Denn ich vermute wie du, dass es auch eine reformiert beziehungsweise protestantisch geprägte Kunst gibt, die sich von der katholisch geprägten in manchem unterscheidet.

David Plüss: Ich schlage vor, dass wir uns zwei zeitgenössische Werke vornehmen, die jüngst für zwei Schweizer Kirchen entstanden sind. Zweimal Glasfenster. Die einen hat Judith Albert in Zusammenarbeit mit Gery Hofer 2008 bis 2010 für die katholische Kirche Sacré-Cœur in Montreux geschaffen, die anderen Claudia und Julia Müller 2010 für die reformierte Kirche in Pratteln bei Basel. Albert ist katholisch, die Geschwister Müller sind in einem reformierten Pfarrhaus aufgewachsen. ${ }^{4}$

\section{Der Gegenstand unseres Gesprächs}

Johannes Stückelberger: Die Voraussetzungen waren an beiden Orten ähnlich. Die Fenster im Chor der reformierten Kirche in Pratteln zeigen eine Farbkomposition, die sich über alle fünf Fenster erstreckt, die folglich wie ein einziges Bild gelesen werden kann, als Landschaft mit einer großen roten Wolke. In diese Farblandschaft hinein haben die Künstlerinnen mit Schwarzlot sieben, aus der Ferne kaum erkennbare Motive gezeichnet: ein nacktes Menschenpaar, ein Huhn mit drei Küken, ein Ei, Werkzeuge, dreimal ein X, eine Flöte, eine Schachtel mit einem Totenkopf darin sowie eine Blumenwiese. Für die Fenster verwendeten die Künstlerinnen Antikgläser, die jedoch nicht in der traditionellen Technik der Bleiverglasung gefasst, sondern auf ein Trägerglas aufgeklebt sind..$^{5}$

3 Vgl. Stückelberger, Johannes: Gottesbilder in der modernen Kunst, in: Gott sehen. Das Überirdische als Thema der zeitgenössischen Kunst, Ausstellungskatalog Warth (Kunstmuseum des Kantons Thurgau), Sulgen/Zürich (Niggli) 2005, S. 43-49.

4 Aktuelle Biografien und Publikationslisten der drei Künstlerinnen in: www.judithal bert.ch; http://www.peterkilchmann.com/artists/ (beide ges. am 24.4.2012).

$5 \mathrm{Zu}$ den Fenstern von Claudia und Julia Müller in Pratteln: Die Glasfenster von Claudia und Julia Müller in der reformierten Kirche Pratteln, hg. von Clara Moser, Brassel, Claudia und Julia Müller, Johannes Stückelberger, Pratteln: Reformierte Kirchgemeinde 
In Montreux galt es Fenster zu ersetzen, die im Juli 2005 durch einen Hagelsturm zerstört worden waren. Albert übernahm von diesen Vorgängerscheiben das Motiv der Heiligenfiguren, die nun - auf ihre Umrisse reduziert - vor einem prächtigen Abendhimmel erscheinen. Die Künstlerin verwendete Fotografien realer Himmel als Vorlagen, die sie in einer neuartigen Technik mit Glaskeramikfarben transluzid auf Antikglas übertrug. ${ }^{6}$ Beide Arbeiten folgen insofern einer ähnlichen Anlage, als in beiden der Himmel eine dominante Rolle spielt.?

\section{HimmeL}

Johannes Stückelberger: Zwischen den beiden Arbeiten gibt es einen kleinen, jedoch entscheidenden Unterschied. In Pratteln zeigt sich unter dem Himmel ein schmaler Streifen Landschaft, der in den Fenstern in Montreux fehlt. Dort sind wir eingeladen, in die Ferne zu schauen und mit unseren Augen umherzuschweifen. In Pratteln bewirkt der Horizont, dass wir die Wolke in einer Beziehung zur Erde und damit auch zu unserem eigenen Standort wahrnehmen.

Im Kontext der Kirche liegt es nahe, eine Himmelsdarstellung mit Vorstellungen des religiösen Himmels, wenn nicht sogar mit der Vorstellung von Gott in Verbindung zu bringen. So darf man auch die Arbeiten von Albert und Claudia und Julia Müller daraufhin befragen, wie sie den Himmel interpretieren, welche Vorstellungen von Gott sie vermitteln. Landschaftsdarstellungen als Gottesbilder zu lesen, dafür gibt es seit der Romantik eine Tradition. So deutet Caspar David Friedrich etwa die untergehende Sonne in seinem Gemälde Kreuz im Gebirge als Metapher für Gott, als ,Bild des ewigen, allbelebenden Vaters“. Eines Gottes, den wir direkt nicht sehen können, dessen Strahlen jedoch auf ihn verweisen und die im Bild die Wolken rot färben. ${ }^{8}$ Die Arbeiten in Montreux und Pratteln stehen in dieser Tradition.

Pratteln-Augst, 2010 (mit Beiträgen von Moser, Clara, Stückelberger, Johannes, Derix, Barbara und René Salathé), Stückelberger, Johannes: Neue Kirchenfenster in der Reformierten Kirche Pratteln/Schweiz, in: Kunst und Kirche, 4, 2010, S. 64-65.

$6 \mathrm{Zu}$ den Fenstern von Judith Albert und Gery Hofer in Montreux: Les nouveaux vitraux du Sacré-Coeur à Montreux de Judith Albert und Gery Hofer, s.l., s.d. [mit Beiträgen von Françoise Jaunin und Stefan Trümpler]; http://www.judithalbert.ch/pdf/works.pdf (ges. am 12.12.2011).

7 Ausführlicher habe ich mich mit Darstellungen und Deutungen des Himmels in der Moderne befasst in: Stückelberger, Johannes: Wolkenbilder. Deutungen des Himmels in der Moderne, München (Wilhelm Fink) 2010 (Habilitationsschrift, Basel 2003).

8 Aus einem Brief von Caspar David Friedrich an Professor Schulz, Dresden, 8. Februar 1809, hier zitiert nach: Uerlings, Herbert (Hg.): Theorie der Romantik, Stuttgart (Reclam), 2000, S. 279. Zu Friedrichs Bild Kreuz im Gebirge (auch „Tetschener Altar“ 



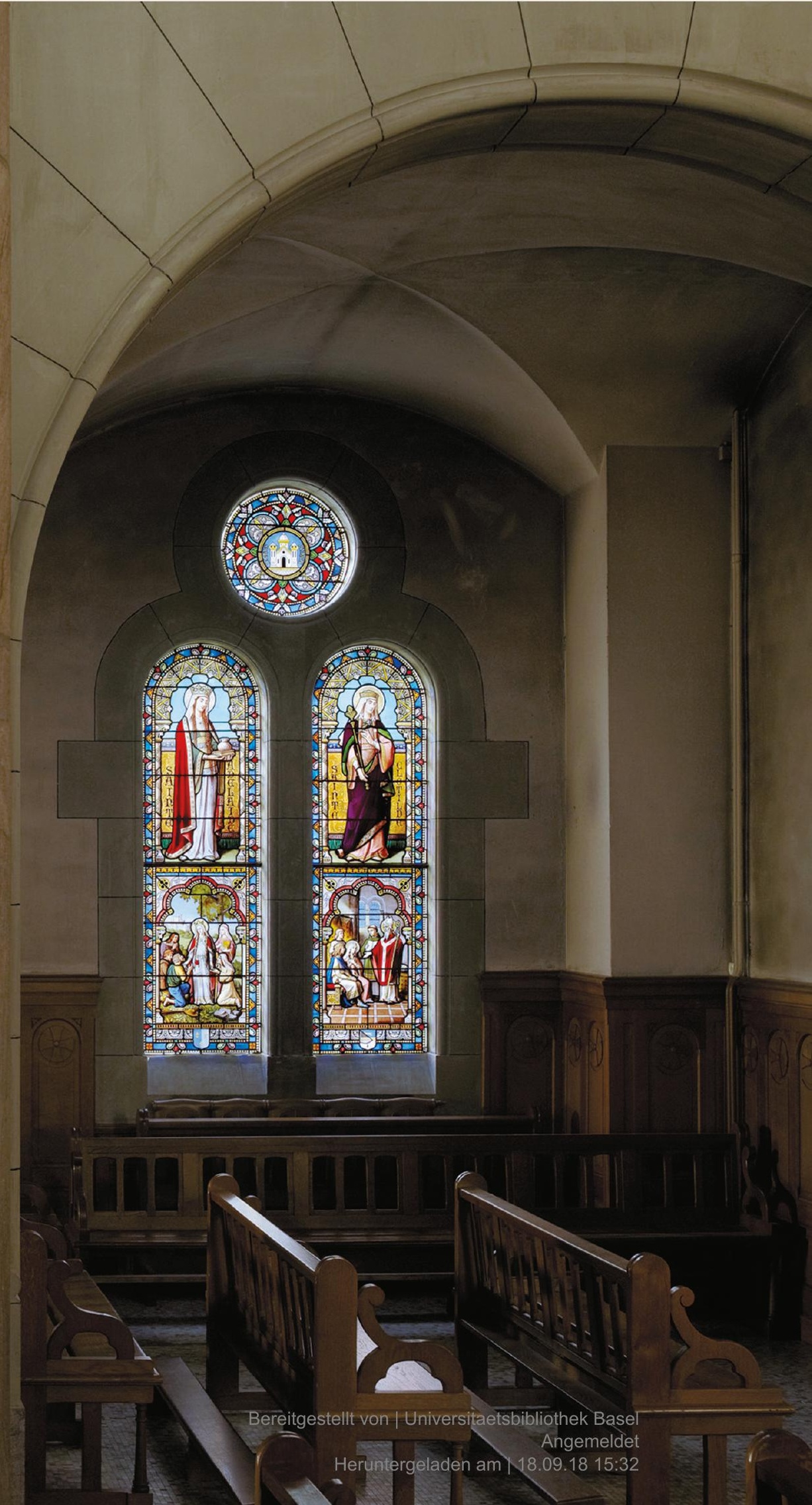


In Montreux schaue ich durch die Fenster hindurch in einen Außenraum, auf Wolken, die sich in einer nicht näher bestimmbaren Distanz zu uns befinden. $\mathrm{Zu}$ dieser Wahrnehmung tragen nicht unwesentlich die Heiligen bei, die auf der Schwelle zwischen dem Innen- und dem Außenraum zu stehen scheinen, gleichsam in die waagrechten Windeisen ,eingewoben ' beziehungsweise auf ihnen stehend. Die Windeisen und die Heiligen sind - einem Spinnennetz nicht unähnlich - im Fensterrahmen verankert und markieren die Ebene der Fensteröffnung. In Pratteln nimmt man die Fensterfläche anders wahr. Sie ist nicht die transparente Membran, die Innen und Außen trennt, vielmehr hat man den Eindruck, die Landschaft und insbesondere die rote Wolke klebten von außen auf den Scheiben. Dadurch und durch ihre Größe erscheint die Wolke sehr nahe.

In Montreux schauen wir von hier nach dort, von innen nach außen. Auf der Schwelle stehen die Heiligen. In Pratteln befindet sich auf dieser Schwelle die Wolke. Die Fenster von Claudia und Julia Müller wirken nicht durchsichtig, was die in Montreux natürlich auch nicht sind, aber die dortigen Motive des Wolkenhimmels und der Heiligen suggerieren doch diese Wahrnehmung. Die Wolke in Pratteln macht auf mich den Eindruck, als wolle sie sich ins Kircheninnere ergießen. Anders formuliert: In Montreux schauen wir den Himmel an, in Pratteln schaut der Himmel uns an. In beiden Arbeiten geht es um die Darstellung religiöser Erfahrung. In Montreux ist es die Erfahrung von Transzendenz (Gott als der Andere), in Pratteln die Erfahrung von Immanenz (Gott im Eigenen).

$\mathrm{Zu}$ diesen unterschiedlichen Auffassungen von Gottesschau lassen sich Parallelen ausmachen in der Theologie: Der Protestant Friedrich Schleiermacher definiert Religion als Anschauung des Unendlichen im Endlichen. Religiöse Erfahrung ist für ihn - so lese ich ihn - eine Erfahrung von Transzendenz in der Immanenz, die Erfahrung, dass Gott in unsere Wirklichkeit hineinwirkt. So, wenn er etwa von der „Vermählung des Unendlichen mit dem Endlichen“ spricht oder davon, dass sich ihm „unter dem Endlichen und Einzelnen das Bewusstsein des Unendlichen und des Ganzen“ entwickelt hat.9 Zu meiner Formulierung, der Himmel in Pratteln schaue uns an, gibt es bei Schleiermacher eine schöne Parallele, wobei ich nicht ausschließe, dass meine Deutung der Pratteler Fenster von meiner Schleiermacher-Lektüre beeinflusst ist. Im Zusammenhang mit Überlegungen zur Anschauung des Universums schreibt der deutsche Theologe: „Alles Anschauen gehet aus von einem Einfluss des Angeschaueten auf den Anschauenden, von einem ursprünglichen und unabhängigen Handeln des ersteren, welches dann von dem letzteren seiner Natur

genannt) von $1807 / 08$ vgl. Busch, Werner, Caspar David Friedrich. Ästhetik und Religion, München (Beck) 2003, S. 34-45.

9 Schleiermacher, Friedrich Daniel Ernst: Über die Religion. Reden an die Gebildeten unter ihren Verächtern [1799], Hamburg (Reimer) 1958, S. 149. 
gemäß aufgenommen, zusammengefasst und begriffen wird. “ ${ }^{10}$ Religiöse Erfahrung stellt sich nach Schleiermacher so ein, dass das Jenseits ins Diesseits ,hineinscheint'.

Anders sieht der katholische Theologe Günter Rombold das Verhältnis von Transzendenz und Immanenz. Die durch Kunst vermittelte religiöse Erfahrung ist für ihn eine Erfahrung des Überschreitens einer Schwelle in eine andere Wirklichkeit. Transzendenzerfahrung stellt sich für Rombold so ein, dass wir vom Hier in ein Dort hinüberschauen. So schreibt er: „Wir verstehen darunter [unter Transzendieren, Anm. J. S.] das Überschreiten der endlichen Wirklichkeit auf die unendliche hin, der bedingten Wirklichkeit auf die unbedingte, der relativen auf die absolute. “Wobei, so schließt er an, „zu bemerken ist, dass dabei das eine auf das andere bezogen bleibt: Das Unbedingte wird im Bedingten gefunden, das Absolute im Relativen. “ ${ }^{11}$

David Plüss: Die von dir vorgenommene Beschreibung und Kontextualiserung der beiden Werke ist hilfreich. Der These, wonach sich der Protestantismus programmatisch auf das Diesseits bezieht, stimme ich grundsätzlich zu. Dies gilt zumindest seit dem 19. Jahrhundert. Schleiermacher, auch Kirchenvater des Neuprotestantismus genannt, hat allerdings, wie du selber zeigst, keine platte Diesseitigkeit von Religion vertreten, sondern gerade auf die unaufhebbare Spannung von Immanenz und Transzendenz Wert gelegt. Religion ist für ihn bekanntlich „weder Denken noch Handeln, sondern Anschauung und Gefühl“, nämlich das Gefühl schlechthinniger Abhängigkeit, „Sinn und Geschmack fürs Unendliche“. ${ }^{12}$ Religion wird vom jungen Schleiermacher geradezu ästhetisiert. Gefühl und Anschauung machen das Religiöse als solches aus, nicht aber das sittliche Handeln im Alltag oder die Vernunft. ${ }^{13}$ Diese Ästhetisierung ermöglicht ihm, die Transzendenz zu denken und sie vor allem sinnlich zu erfahren. Der Immanenzbezug wird dabei an keiner Stelle aufgegeben, sondern stellt den Zielpunkt religiöser Transzendenzerfahrung dar. Religion ist immer eine solche des religiösen Subjekts und auf dessen religiöse Selbstentfaltung im Diesseits

10 Ebenda, S. 31.

11 Rombold, Günther: Transzendenz in der modernen Kunst, in: Schmied, Wieland (Hg.): Zeichen des Glaubens - Geist der Avantgarde: religiöse Tendenzen in der Kunst des 20. Jahrhunderts, Ausstellungskatalog Berlin (Schloss Charlottenburg, Grosse Orangerie), Stuttgart/Mailand (Klett-Cotta/Electa) 1980, S. 14-26, hier S. 15. - Vgl. Rombold, Günther: Das Transzendieren des Kunstwerks, in: ders.: Der Streit um das Bild. Zum Verhältnis von moderner Kunst und Religion, Stuttgart (Katholisches Bibelwerk), 1988, S. 261-268.

12 Schleiermacher 1958 (wie Anm. 9), S. 29-30.

13 Vgl. Safranski, Rüdiger: Romantik. Eine deutsche Affäre, München (Carl Hanser) 2007 , S. 145 . 
bezogen. Dieses Religionsmodell kann als typisch (neu-)protestantisch gelten und scheint mir auch für eine Dechiffrierung konfessioneller Konturen der Gegenwartskunst hilfreich.

Die Glasfenster von Montreux metaphorisieren tatsächlich ein Jenseits. Sie entwickeln einen machtvollen Sog in das Reich der Wolken und den mit mythischen Heiligenfiguren bevölkerten Himmel. Aber sie tun dies mittels einer fast schon überzeichneten Realistik der Wolken und der Farben. Die Fenster in Pratteln dagegen verblüffen mit einer knallrot-gelben Großwolke, die einen nicht wegzieht, sondern geradezu bedrängt und die Selbstreflexion des betrachtenden Subjekts provoziert. Die Subjektivität ästhetischer Wahrnehmung und sinnlicher Erfahrung ist hier unvermeidbar - und damit der Diesseitsbezug von Religion. Mein Fazit: Sowohl die Pratteler Glasfenster als auch diejenigen in Montreux eröffnen die Spannung zwischen Immanenz und Transzendenz, allerdings mit unterschiedlichen Ankerpunkten. Während der Abendhimmel Judith Alberts die Frömmigkeit im Jenseits verankert, haftet die durch die Glasfenster von Claudia \& Julia Müller evozierte Religion am religiös affizierten Subjekt.

\section{TraditION}

Johannes Stückelberger: Aufschlussreich für die Frage der konfessionellen Prägung scheinen mir auch die den beiden Wettbewerben vorausgegangenen Programme zu sein. Teil der Ausschreibung in Montreux war eine „Message de la Paroisse“, in der die Pfarrei ihre Erwartungen formulierte. Erstens sollen die Scheiben als Zeugnisse der Kunst unserer Zeit erkennbar und gleichzeitig der Tradition verpflichtet sein. Zweitens sollen sich die Wettbewerbsteilnehmer/ innen von den traditionellen alten Scheiben inspirieren lassen. Und drittens sollen die Fenster an die traditionelle Aufgabe der Kirche erinnern, die darin besteht, das Reich Christi zu verkünden. ${ }^{14}$ Dreimal ist von Tradition beziehungsweise von der Tradition der Kirche die Rede. Das lässt mich fragen - und diese Frage möchte ich an dich richten -, worin die Protestanten die Aufgabe der Kirche sehen und welche Rolle bei ihnen die Tradition spielt.

David Plüss: Tatsächlich unterscheiden sich Katholiken und Protestanten in ihrem jeweiligen Traditionsbezug beträchtlich. Während für die römisch-katholische Kirche die Lehrentscheidungen der Konzilien und die ,ex-cathedra'-

14 Concours de projets pour la recréation de cinq vitraux (détruits par la grêle du 18.7.2005 et irréparables) à l'église catholique du Sacré-Cœur, avenue des Planches 27, 1820 Montreux, Annexe Vo2 (du règlement du premier tour): Message de la Paroisse, 2007 (Typoskript), Archiv Johannes Stückelberger, Therwil. 
Entscheide des Papstes normative Geltung haben und Unfehlbarkeit für sich reklamieren, lehnen die Protestanten diesen Anspruch sowie viele einzelne Entscheidungen ab. ,Sola scriptura', einzig die Bibel kann für sie Maßstab sein. Oder genauer: einzig das durch die Auslegung des Bibeltextes in der jeweiligen Gegenwart verkündigte und den Menschen befreiende Gotteswort. Es wird ein vermeintlich traditionsfreier Rückgriff auf die Heilige Schrift postuliert und zudem die Unmittelbarkeit göttlicher Erleuchtung durch den Heiligen Geist im Prozess des Auslegens und Verstehens. Die theologische Hochschätzung der Tradition dagegen ist Protestanten fremd und suspekt. Ich meine, dass sich dieser Unterschied auch beim Vergleich der beiden Werke zeigt. Die durch Silhouetten angedeuteten Heiligen, welche in Montreux gleichsam als Mittler und Schwellenwesen fungieren, wären in Pratteln undenkbar.

Johannes Stückelberger: In Pratteln haben Claudia und Julia Müller sieben unterschiedliche Motive auf die Scheiben gezeichnet. Drei davon kennen wir aus der christlichen Ikonografie: das Menschenpaar, den Totenschädel sowie das $\mathrm{X}$, wobei letzteres - im griechischen Alphabet ist es der Buchstabe „Chi“ - für Christus steht. Die übrigen Motive zählen nicht zu den bekannten kirchlichen Symbolen. Man fragt sich, wie sie mit biblischen oder theologischen Inhalten in Zusammenhang gebracht werden können. Dazu ein paar Hinweise: Die Zeichen verweisen auf Stationen des Lebens von der Geburt (Ei), über die Ehe (Paar) bis zum Tod (Totenkopf): Stationen, die in der Kirche mit Übergangsritualen wie Taufe, Hochzeit oder Beerdigung gefeiert werden. Sie stellen etwa die Frage, was zuerst war, das Huhn oder das Ei. Sie versinnbildlichen in der Blumenwiese den unermesslichen Reichtum und die Fülle der Schöpfung. Sie verweisen mit den Werkzeugen auf das tätige Leben und mit der Flöte auf die Kunst. Und dies alles ist einer Landschaft eingeschrieben, die von Gottes Präsenz durchwirkt ist. Das menschliche Leben und Tun wird in den Pratteler Scheiben in eine Beziehung gestellt zu Gott. Die Kirche wird als Ort definiert, an dem man sich immer wieder neu dieser Beziehung versichert, sie lebt und bekennt.

Die Künstlerinnen sprechen von den Zeichen als visuellem Vokabular, das sich mit Symboltraditionen verbinden lasse, das jedoch immer auch individuell bestimmbar bleiben und poetische Momente evozieren soll. ${ }^{15}$ Mit dieser Aussage und mit ihrer ,neuen‘ Symbolsprache laden sie zu einer persönlichen Auseinandersetzung mit den ganz alten und gleichzeitig aktuell bleibenden theologischen Fragestellungen ein. Sie sagen damit: Die biblischen Inhalte müssen immer wieder neu interpretiert werden, die Kirche muss sich immer wieder

15 Claudia und Julia Müller: Suche nach Sieben. Wettbewerb für neue Kirchenfenster im Chor der Reformierten Kirche Pratteln, Dezember 2008 (Typoskript), Archiv Johannes Stückelberger, Therwil. 
erneuern, gemäß dem Wahlspruch der Reformierten: „Ecclesia reformata et semper reformanda“.

David Plüss: Ich habe vorhin vom vermeintlich traditionsfreien Rückgriff auf die in den Heiligen Schriften offenbarte göttliche Wahrheit gesprochen. Tatsächlich ist ein solch direkter, unverstellter Rückgriff nicht möglich. Auch die protestantische Religion ist eine traditionsvermittelte. Auch wir Protestanten stehen in einem kulturellen Gedächtnisraum und pflegen diesen in Theologie, Kirche und Gesellschaft. Eine unterstellte Unmittelbarkeit ist naiv und sogar gefährlich. Insofern vermisse ich bei uns Protestanten zuweilen den ästhetischen, spirituellen und reflexiven Umgang mit Tradition, wie er für Katholiken selbstverständlich ist. Protestantinnen und Protestanten sind oft erstaunlich ahnungslos, was ihre eigene Herkunft und Tradition anbelangt - eine Tradition, die wir nota bene über 1'500 Jahre miteinander teilen. Die in Pratteln verwendeten Motive scheinen mir symptomatisch für diesen mehrheitlich ignorierten oder durchgestrichenen Traditionsbezug des Protestantismus. Sie sind diskret, man sieht sie kaum. Sie regen die Reflexion an, gewiss, aber sie versetzen uns nicht in einen durch religiöse Motive eröffneten Andachtsraum. Christentum und Glaube werden nicht unmittelbar thematisch, sie werden nicht symbolisiert. Die religiöse Konnotation der Motive ergibt sich in durchaus produktiver Offenheit durch den Kirchenraum. Das ist für das religiöse Individuum reizvoll und anregend, aber in Bezug auf die eigene Tradition und Ikonografie auch ein wenig verschämt.

\section{KIRCHE}

Johannes Stückelberger: Den beiden Arbeiten in Pratteln und Montreux liegt - so möchte ich behaupten - ein unterschiedliches Verständnis von Kirche zugrunde. Albert beschließt ihre Projekteingabe mit einer allgemeinen Bemerkung zur Rolle der Glasfenster in einer Kirche als Kontaktstelle zwischen dem heiligen Raum und der Quelle, die diesen erhellt. Mit ihrer Arbeit wolle sie zum Ausdruck bringen, dass die Kirche ein Ort sei, an dem Grenzen überschritten werden: Grenzen zwischen Innen und Außen, Tag und Nacht und vor allem zwischen dem Diesseits und dem Jenseits. ${ }^{16}$ Kirche ist für Albert ein Ort der Versöhnung von Diesseits und Jenseits, ein Ort der Transzendenz. Ist das ,typisch katholisch'? Und wenn ja, wie sieht im Unterschied dazu das Kirchenverständnis der Reformierten aus?

16 Judith Albert: Ciel. Démarche créatrice/Descriptif technique. Un projet de Judith Albert en collaboration avec Gery Hofer, 2008 (Typoskript), Archiv Johannes Stückelberger, Therwil. 
David Plüss: Die Kirche im katholischen Verständnis ist ein Sakralraum, und zwar sowohl im theologischen („extra ecclesia non salus est“) als auch im räumlichen Sinn. Katholische Kirchen sind geweihte Orte mit geheiligten Elementen - geweihtes Wasser beim Eingang, gewandelte Hostien im Tabernakel, verehrte Altarreliquien. Das Heilige ist gleichsam physisch präsent und sinnlich wahrnehmbar. Das Gebet und die Frömmigkeit haben ihre Kraftorte und ihre Ausrichtung. Wenn nach Albert die Kirchenfenster zwischen Sakralraum und Transzendenz vermitteln, so handelt es sich hier um eine prägnant katholische Funktionszuweisung. - Im protestantischen Verständnis sind Kirchenräume dagegen weitgehend pragmatisch bestimmt. Sie haben bestimmte Funktionen zu erfüllen: Sie haben der Versammlung der Gemeinde, der frommen Belehrung und der Andacht zu dienen. Ihre sakrale Qualität bemisst sich daran, ob und in welcher Weise sie diese Funktionen erfüllen. Und auch wenn sie für die Feiernden einen Schutz- und Freiheitsraum darstellen, so befindet sich dieser doch ganz in der Welt und grenzt an kein Jenseits. Kirche ist für Protestanten zwar ein visionäres Projekt, aber kein Himmel auf Erden. Auch dies wird in Pratteln schön sichtbar.

\section{GotT}

Johannes Stückelberger: Ich habe oben das Programm des Wettbewerbs von Montreux erwähnt. Auch in Pratteln hat die Kirchgemeinde ein Programm formuliert. An einem Gesprächsabend, zu dem alle Mitglieder der Gemeinde eingeladen waren, haben sich die Anwesenden über ihre Gottesbilder ausgetauscht und darüber, was ihnen Kirche bedeutet. Die Gedanken wurden in dem Satz zusammengefasst, der dann auch dem Wettbewerbsprogramm als Grundlage diente: „Gott lädt uns ein, ihm nahe zu sein. Diese göttliche Kraft stärkt uns, einander über Grenzen hinweg zu begegnen. “ ${ }^{17}$ Dem Satz liegt - so scheint mir - ein anderes Gottesbild und auch ein anderes Verständnis von Kirche zugrunde als dem Programm von Montreux. Doch was ist es, das uns diesen Satz als protestantische Formulierung empfinden lässt?

David Plüss: Gott wird hier als Kraft bestimmt, die in zwischenmenschlichen Beziehungen Gestalt gewinnt. „Gott als Kraft der Beziehung“, wie es die amerika-

17 Protokoll des Gesprächsabends mit Interessierten der Gemeinde am 10. April 2008. Anhang 1 zu: Evang.-ref. Kirchgemeinde Pratteln-Augst, Wettbewerb für neue Kirchenfenster im Chor der Reformierten Kirche Pratteln. Wettbewerbsprogramm, von der Jury genehmigt am 25. Juni 2008 (Typoskript), Archiv Johannes Stückelberger, Therwil. 
nische Feministin Carter Heyward prägnant formuliert hat. ${ }^{18}$ Räume, Symbole und geweihte Ämter spielen hier zunächst keine Rolle. Die Gotteskraft bedarf keiner Vermittlung, sondern wirkt unmittelbar und bewährt sich in Mitmenschlichkeit und Verantwortung. Dass sich Protestantinnen und Protestanten mit dieser Unmittelbarkeit, Diesseitigkeit und Verantwortung auch immer wieder schwer tun und überfordern, ist die Rückseite derselben Medaille. Dass religiöse Sinnsuche und Selbstvergewisserung Schutz- und Kraftorte braucht, Orte der Berührung und der Verwandlung, Orte der Schönheit und der Erbauung, Orte des Geheimnisses und der Absolution haben die Katholiken deutlicher erkannt als wir Protestanten. Gleichwohl bleibt die in Pratteln sinnlich gestaltete Religion nicht im Diesseits des Zwischenmenschlichen stecken, sondern eröffnet eine Atmosphäre der Andacht, einen weiten, transzendenzoffenen Raum.

Johannes Stückelberger: Auch in den Pratteler Fenstern wird eine direkte Gottesbeziehung thematisiert. Die im Programm formulierte „Einladung Gottes, ihm nahe zu sein“, bringe ich mit der roten Wolke in Verbindung, die sich von rechts in den Kirchenraum schiebt. Die Leserichtung in unserer Kultur verläuft von links nach rechts. Die Wolke ist gegenläufig, was ich als Metapher dafür lese, dass Gott uns entgegenkommt. In den Fenstern durchdringen sich zwei Bewegungen. Die eine folgt dem Lauf der Sonne von links nach rechts: das Nordfenster ist in blauen Tönen gehalten, die für die Nacht stehen, vom Ost- zum Südfenster gibt es eine steigende Bewegung im grünen Bodenstreifen, was man als Zeichen für den sich entfaltenden Tag lesen kann. Die andere Bewegung ist wie gesagt die der Wolke von rechts nach links, eine antizyklische Bewegung: Zeichen für das Sich-uns-Zuwenden Gottes.

David Plüss: Das ist eine sehr schöne und einleuchtende Lesart! Allerdings vermag ich hier keine konfessionellen Eigenheiten zu erkennen, sondern die Bebilderung des religiösen Transzendenzbezugs schlechthin.

\section{BILD}

David Plüss: Mich fasziniert der Himmel in der Kirche von Montreux. Er entwickelt eine Sogkraft, die einen hineinzieht - in die Andacht, ins Gebet. Dabei wird mir das ambivalente Verhältnis von uns Protestanten zum religiösen Bild, zur Bebilderung unserer Religion bewusst. Denn der Protestantismus ist eine Religion der Innerlichkeit, vor allem in seiner reformiert-zwinglianischen Spiel-

18 Vgl. Heyward, Carter: Our passion for justice. Images of power, sexuality, and liberation, Cleveland (The Pilgrim Press), 1994. Heyward war Theologieprofessorin an der Episcopal Divinity School in Cambridge (Massachusetts, USA). 
form. Hör- und Sichtbares im Kirchenraum stellt für die Zürcher Reformatoren eine Gefahr dar, weil es vom Eigentlichen ablenkt und zum Götzendienst verführt, sofern nicht Gott, sondern menschliches Machwerk verehrt wird. ${ }^{19}$ Religion soll das Herz des Menschen betreffen und bewegen. Seine Andacht ist der Zielpunkt jeglicher Religionspraxis. Was diese befördert, ist geistlich, was sie hindert, gilt es zu unterlassen oder zu entfernen. Entsprechend wurden die Kirchen geräumt und die Liturgie entschlackt. Reformierte Liturgie ist ein Kult der Innerlichkeit und der entleerten Kirchenräume. Dies ist seit Pietismus, Romantik und Schleiermacher noch verstärkt der Fall.

Dieses spannungsvolle Verhältnis zur Äußerlichkeit bildhafter Darstellung ist auch den Pratteler Kirchenfenstern anzumerken. Die Darstellung ist gegenständlich und zugleich eigenartig abstrakt. Formen und Farben verfremden das Bekannte. Dadurch regen sie die Innerlichkeit des religiösen Erlebens und Reflektierens an und machen sie unabdingbar. Anders die Fenster in Montreux. Sie entlasten den Betrachter von aufwändiger Reflexion und lassen ihn die Schönheit des dargestellten Abendhimmels meditieren und darin das Jenseits erahnen. Das Verhältnis zur bildhaften Darstellung erscheint entspannter und selbstverständlicher. Katholische Frömmigkeit erweist sich hier allerdings keineswegs als bloß äußerliche und gegenständliche. Vielmehr symbolisieren die Darstellungen von Heiligen und Abendhimmel das nicht darstellbare göttliche Geheimnis.

Johannes Stückelberger: Du benennst ganz wesentliche Unterschiede zwischen den beiden Arbeiten, die ich abschließend mit einer unterschiedlichen Auffassung der Funktion von Bildern in Verbindung bringen möchte. Diese unterschiedliche Auffassung ließe sich anhand der Geschichte der katholischen und protestantischen Bildproduktion diskutieren, wofür hier nicht der Platz ist. Ich will mich auf ein paar Hinweise beschränken, die sich auf die Fenster von Pratteln und Montreux beziehen. Nach katholischer Auffassung haben religiöse Bilder und insbesondere Bilder in Kirchen die Funktion von Andachtsbildern, von Bildern, die die Gläubigen zur Andacht hinführen, die sie emotional ansprechen wollen. Im Lauf der Geschichte sind dafür eigene Bildtypen entstanden wie der Schmerzensmann, die Christus-Johannes-Gruppe oder das Heilige Grab. Insbesondere erfüllen auch alle Mutter Gottes- und Heiligendarstellungen diese Funktion: als direkte Gegenüber, zu denen man seine Nöte und Sorgen bringen kann, als Vorbilder, die durch ihr eigenes Leben und Leiden, in das zu vertiefen die Bilder einladen, Trost spenden, oder als Fürbitter, von denen man sich Hilfe erhofft. Die Beziehung zu diesen ,heiligen` Bildern spielt sich

19 Knellwolf, Ulrich: Die Musik im reformierten Gemeindegottesdienst, in: Musik der evangelisch-reformierten Kirche. Eine Standortbestimmung, Zürich (Institut für Kirchenmusik der evangelisch-reformierten Landeskirche des Kantons Zürich) 1989, S. 45-86. 
auf einer emotionalen Ebene ab. Das, denke ich, ist auch die Faszination, die wie du sie beschreibst - von den Fenstern von Albert ausgeht.

Im Unterschied dazu ist die Funktion der Bilder im Protestantismus primär eine hermeneutische. Die Bilder dienen der Schriftauslegung und der Vermittlung von Glaubensinhalten. Sie erzählen, interpretieren und führen in Symbolen vor Augen, woran die Protestanten glauben. Sie übernehmen gleichsam die Rolle von Glaubensbekenntnissen. Und dafür eignet sich eine figürliche Bildsprache besser als eine abstrakte. Die Bilder sollen lesbar sein, wie die Schrift. Bezeichnenderweise haben im 20. Jahrhundert abstrakte Kunstwerke in reformierte Kirchen weit weniger Eingang gefunden als in katholische. Abstrakte Kunst ist den Protestanten suspekt: als Kunst, die nicht direkt auf das Wort verweist, die unmittelbar anspricht und emotional berührt.

Auf einen ersten Blick möchte man vermuten, die Fenster von Judith Albert würden in ihrer auf Fotografien basierenden Gegenständlichkeit und Naturalistik eher einem reformierten, textgebundenen Bildbegriff entsprechen. Dem ist aber nicht so. Die Wolken entziehen sich als Motiv der Textnähe. Natürlich kann man sie als Metaphern für Gott und Unendlichkeit lesen. Doch zunächst berühren sie uns auf einer anderen Ebene. Der gefärbte Abendhimmel versetzt uns ins Staunen, er zieht unseren Blick in die Tiefe, er bringt in uns Saiten zum Schwingen, er berührt uns emotional. Wolkenhimmel haben mit abstrakten Bildern gemein, dass sie unsere Blicke schweifen lassen, dass sie uns nicht auf dieses oder jenes Detail fokussieren, dass sie vielmehr eine Stimmung vermitteln und eine Atmosphäre schaffen. Mit ihren Wolkenhimmeln und den Heiligen funktionieren die Fenster in Montreux wie Andachtsbilder.

Die Fenster in Pratteln wirken zwar auf den ersten Blick und aus der Distanz abstrakter als die in Montreux. Doch laden sie nicht in gleicher Weise wie die letzteren zur Andacht ein. Die Farben sind dafür zu intensiv und zu aktiv, die rot-gelbe Wolke zu groß und zu präsent. Die in die Farblandschaft eingeschriebenen Zeichnungen verlangen vollends eine andere Lektüre. Wir sind aufgefordert, nach den sieben Motiven zu suchen, sie zu deuten und mit uns und unserem Glauben in Beziehung zu bringen. Dabei sind unser Intellekt und unser Assoziationsvermögen gefragt. Nichts von Beschaulichkeit und Andacht. Die Pratteler Fenster sind nicht einfach schön. Sie haben - auch in der Darstellung der Wolke - etwas Sperriges, Widerständiges, Unbequemes, Direktes, das ich mit dem Protestantismus in Verbindung bringe. 


\section{ScHLUSS}

David Plüss: Mir ist aus unserem Gespräch Folgendes deutlich geworden: Die Konfessionalität ist auch in der Gegenwartskunst noch immer erstaunlich stilprägend, zumindest in religiösen Kontexten. Die beiden Kunstwerke von Montreux und Pratteln spiegeln den Frömmigkeitsstil, den Habitus und die Theologien der unterschiedlichen Religionskulturen in eindrücklicher Weise. Zugleich beschränken sich unsere Analysen zunächst auf die beiden Werke, und es wäre durch weitere Vergleiche zu prüfen, ob und in welcher Weise sie eine darüber hinausreichende Allgemeinheit beanspruchen können.

Johannes Stückelberger: Wir haben den Fokus auf die Konfessionalität gerichtet. Dass sie nicht das einzige Element ist, das die von uns diskutierten Werke prägt, versteht sich von selbst. Auch Zeit, Ort, Milieu, Alter, Individualität und anderes tragen zur Ausbildung einer künstlerischen Sprache bei. Und auch die Auftraggeber und die Orte, für die die Fenster geschaffen wurden, spielten in diesem Fall eine Rolle. Und doch dürfte es kein Zufall sein, dass den Wettbewerb in Montreux eine katholisch sozialisierte Künstlerin aus der Innerschweiz gewonnen hat, während beim Wettbewerb in Pratteln zwei reformierte Künstlerinnen zum Zuge kamen. Die wichtigste Voraussetzung war natürlich die künstlerische Qualität der Arbeiten. Gleichwohl: Albert hat offensichtlich am besten gespürt, was zur katholischen Kirche in Montreux passt, während die Schwestern Müller mit ihrem Erfahrungshintergrund in der reformierten Kirche in Pratteln den richtigen Ton fanden. Wobei es natürlich auch Beispiele gibt, wo konfessionslose Künstler oder Angehörige einer anderen Konfession einen kirchlichen Auftrag erhalten, weil es ihnen gelingt, sich in die Aufgabenstellung und die Eigenheiten der jeweiligen Kirche einzufühlen. Darauf hinzuweisen ist mir wichtig, damit nicht der Eindruck entsteht, wir würden alles der konfessionellen Prägung zuschreiben. Und doch ist diese nicht zu unterschätzen, was unser Gespräch deutlich zeigt und was ich in dieser Deutlichkeit nicht erwartet habe. 